\title{
DHEA in women with hypoandrogenism—debate remains open
}

Michał Kunicki, Krzysztof Łukaszuk and Joanna Liss

We read with great interest an article published in the July 2015 issue of Nature Reviews Endocrinology (Genetics of androgen metabolism in women with infertility and hypoandrogenism. Nat. Rev. Endocrinol. 11, 429-441; 2015), ${ }^{1}$ by Aya Shohat-Tal and colleagues. This well-written Review answers, among others, the question of how hormonal profiles of women with hypoandrogenism reflect their ability to metabolize DHEA to testosterone following supplementation with the androgen dehydroepiandrosterone (DHEA). In principle, we agree with their Review; however, we would like to note some points that need to be addressed further.

Shohat-Tal and colleagues state that the benefits of DHEA supplementation might only become clinically apparent in women with testosterone levels below certain (abnormally low) thresholds. However, even though hypoandrogenism is defined as low levels of androgens, what actually constitutes really low levels of testosterone, DHEA and dehydroepiandrosterone sulphate (DHEAS) in each individual patient is not known. We agree that although some women ( $<50$ years of age) have levels of testosterone and DHEAS below $8.68 \mathrm{nmol} / 1$ and $40.74 \mathrm{nmol} / 1$, respectively, these women cannot have hypoandrogenism. ${ }^{2}$ Furthermore, some women with normal ranges of these hormones develop symptoms of hypoandrogenism and can have low functional ovarian reserve (LFOR), especially those women with borderline results. Distinguishing between low-normal and high-normal ranges of these hormones (such as for low-normal and high-normal levels of thyroid-stimulating hormone) might be needed.

The methodology for assessment of androgen levels is important, as was mentioned by Shohat-Tal and colleagues in their introduction. ${ }^{1}$ However, we think that it should be stressed that assessment of levels of testosterone and other androgens should only be performed using tandem mass spectrometry and liquid chromatography. Many studies still present data obtained using older methods such as radioimmunoassay. ${ }^{3}$

We also do not know how long DHEA supplementation should last and no agreement has been reached as to the optimal dosages that should be used (amounts suggested vary between $50 \mathrm{mg}$ and $75 \mathrm{mg}$ per day). ${ }^{4,5}$ The 6-week treatment duration cited by Shohat-Tal and colleagues is probably insufficient, as other reports suggest 3-4 months of DHEA supplementation. ${ }^{6,7}$

Shohat-Tal and colleagues speculated that African-American women would benefit more from supplementation with testosterone than from supplementation with DHEA. We wonder if similar results could be achieved by administering higher dosages (for example, $100 \mathrm{mg}$ per day) of DHEA; however, only parallel studies can definitively answer this question. Conversely, people of other races (for example, people of Chinese descent) who have higher conversion rates of DHEA to testosterone than individuals of African descent could be given lower dosages of DHEA.

Another important issue is outcomes of in vitro fertilization cycles with comprehensive DHEA supplementation in women with LFOR. In our study, performed on 459 women undergoing cycles of intracytoplasmic sperm injection for the first time in a long gonadotropin-releasing hormone agonist protocol, we measured both levels of testosterone and DHEA and/or DHEAS before ovarian stimulation. ${ }^{8}$ We concluded that DHEA and/or DHEAS levels (in addition to known factors of ovarian reserve such as antral follicle count and basal levels of follicle-stimulating hormone and antiMüllerian hormone) do not predict clinical pregnancy. On that basis, we speculated that DHEA supplementation is not effective in women with 'normal' levels of DHEAS. ${ }^{8}$

Finally, the assessment of both cortisol and adrenocorticotropic hormone levels is still not part of a routine work-up in the management or diagnosis of infertility. We believe that the points we have raised here are important for the management of infertility.

INVICTA Fertility and Reproductive Center, ul. Trzy Lipy 3, 80-172 Gdansk, Poland (M.K., J.L.). Department of Obstetrics and Gynecological Nursing, Faculty of Health Sciences, Medical University of Gdansk, ul. Debinki 7, 80-211 Gdansk, Poland (K.L.). Correspondence to: M.K.

mkunicki@op.pl

\section{Competing interests}

The authors declare no competing interests.

1. Shohat-Tal, A., Sen, A., Barad, D. H., Kushnir, V. \& Gleicher, N. Genetics of androgen metabolism in women with infertility and hypoandrogenism. Nat. Rev. Endocrinol. 11, 429-441 (2015).

2. Guay, A. \& Davis, S. R. Testosterone insufficiency in women: fact or fiction? World J. Urol. 20, 106-110 (2007).

3. Janse, F., Tanahatoe, S. J., Eijkemans, M. J. \& Fauser, B. C. Testosterone concentrations, using different assays, in different types of ovarian insufficiency: a systematic review and meta-analysis. Hum. Reprod. Update $\mathbf{1 8}$ 405-419 (2012).

4. Mamas, L. \& Mamas, E. Premature ovarian failure and dehydroepiandrosterone. Fertil. Steril. 91, 644-646 (2009).

5. Gleicher, N., Weghofer, A. \& Barad, D. H. Improvement in diminished ovarian reserve after dehydroepiandrosterone supplementation. Reprod. Biomed. Online 21, 360-365 (2010).

6. Gleicher, N. \& Barad, D. H. Dehydroepiandrosterone (DHEA) supplementation in diminished ovarian reserve (DOR). Reprod. Biol. Endocrinol. 9, 67 (2011).

7. Yeung, T. W., Li, R. H., Lee, V. C., Ho, P. C. \& Ng, E. H. A randomized double-blinded placebo-controlled trial on the effect of dehydroepiandrosterone for 16 weeks on ovarian response markers in women with primary ovarian insufficiency. J. Clin. Endocrinol. Metab. 98, 380-388 (2013).

8. Kunicki, M., Łukaszuk, K., Jakiel, G. \& Liss, J. Serum dehydroepiandrosterone sulphate concentration is not a predictive factor in IVF outcomes before the first cycle of $\mathrm{GnRH}$ agonist administration in women with normal ovarian reserve. PLoS ONE 10, e0118570 (2015). 